\title{
Self-reported alcohol topography, past alcohol use history, and current alcohol cravings
}

\author{
Jonathan K. Noel, PhD, MPH
}

\begin{abstract}
Introduction: Drinking topography is the idea that how a person drinks, in addition to how much they drink, can influence the immediate and long-term effects of alcohol consumption. Measures are often crude, and there has been little effort to associate such measures with disease status.

Methods: To address these gaps, an online survey was completed by 734 U.S. participants. They described how they usually drank using a series of 20 words (e.g., chug, nip, nurse, shots). A latent class analysis was performed to identify clusters of descriptors. Past alcohol use history, as measured by the Alcohol Use Disorders Identification Test (AUDIT), and current alcohol cravings, as measured by the Desires for Alcohol Questionnaire (DAQ), were then regression on class assignment. The analysis was adjusted for age, sex, race, ethnicity, marital status, income, and education level.
\end{abstract}

\section{Introduction}

In the tobacco control literature, topography, the measurement of how one uses a tobacco product, is well defined. These measurements often include number of puffs per cigarette, puff duration, inter-puff interval, and total puff time. Importantly, smoking topography has been associated with toxicant exposure (Pickworth et al., 2017), smoking cessation success rates (Franken et al., 2006), and product characteristics (Hammond et al., 2005). However, the concept of alcohol topography, the measurement of how one consumes an alcoholic beverage, has not been widely described, despite its success in related fields.

Quantitative measurements of alcohol topography are inconsistent. For example, in a study to determine if volumetric information on
Results: Three LCA classes were accepted, with the class characterized by the words shot, swig, gulp, chug, and guz:le having significantly higher AUDIT $\mathrm{b}[95 \%$ $\mathrm{CI}=1.22[0.98,1.46], \mathrm{p}<0.001)$ and DAQ $(\mathrm{b}[95 \%$ $\mathrm{CI}=12.9[10.5,15.3], \mathrm{p}<0.001)$ scores relative to the class characterized by sample, sip, and nurse. The third class predominantly used drink and sip, and was not significantly different from the sample class ( $\mathrm{p}$ 's $=$ 0.290-0.471).

Conclusions: Measuring drinking topography offers a unique way to identify individuals at risk of an alcohol use disorder or those who may be resistant to treatment. It may also be useful in determining drinking motives. However, additional research is needed.

Keywords: alcohol, drinking, topography, cravings, alcohol use disorders

glassware changed consumption times, topography was defined as the total drink time, from the start of the first sip to the end of the last sip (Troy et al., 2017). In a randomized control trial on alcohol self-administration in young adults, topographic measurements included the number of self-administered beers, the inter-drink interval, and drink duration (Leeman et al., 2013). A similarly designed study to determine the effect of automatic action tendency retraining measured average beer drinking duration and inter-drink interval (Leeman et al., 2018). Finally, an ecological momentary assessment study required participants to complete drink reports, where they recorded the total amount of alcohol consumed and the type of beverage involved (Carpenter et al., 2019). Estimated blood alcohol

${ }^{1}$ Correspondence may be sent to: Jonathan K. Noel, PbD, MPH, Department of Health Science, Johnson \& Wales

University, 8 Abbott Park Place, Providence, RI, USA 02903; Tel.: +1 401598 5159; Fax: +1 4015984834 
concentration (BAC) levels were then calculated. More granular alcohol topography measurements, such as total sip duration, total sip interval duration, and number of sips taken, were made via recording and viewing by study staff (Attwood et al., 2012).

Other quantitative topography measures are possible through increased availability of wearable biosensors, which can detect BAC levels based on transdermal sensor information, consumption patterns, and knowledge of an individual's alcohol metabolism (Roberts \& McKee, 2019). Once detected, measurements of peak $\mathrm{BAC}$ and rate of $\mathrm{BAC}$ increase or decrease can be calculated, and cluster analysis has been applied to such data to correlate BAC trajectories with self-reported alcohol use (Gunn et al., 2021). Other wearable biosensors can measure sip number, sip length, and other topographic metrics if users comply with device prompts to indicate when drinking sessions start and the type of beverage consumed.

Despite these initial methodological steps, there are substantial gaps in the alcohol topography literature. Most topographic measurements focus solely on beer consumption while excluding wine and spirits, whose consumption patterns may be inherently different, and it is not clear if true objective measurements are possible since user or investigator input has often been required. Attempts to associate topography with health outcomes largely focus on estimated BAC levels, although one study concluded that peak number of standard drinks was associated with alcohol use disorder (AUD) symptoms (Carpenter et al., 2019). Finally, discussions on the use of wearable biosensors have primarily focused on AUD medication development (Roberts \& McKee, 2019).

In response, the current exploratory study sought to expand the existing alcohol topography literature by measuring qualitative self-reported drinking styles, conducting a latent class analysis on such responses, and then regressing measures of individual alcohol use history and alcohol cravings on the resultant latent classes. It was thought that several unique clusters of drinking styles would emerge and that each cluster would be associated with a unique level of drinking and alcohol craving.

\section{Methods}

Sample Recruitment

A convenience sample of participants were recruited from Amazon's Mechanical Turk (MTurk), which is an online crowd-sourcing service where workers complete tasks for small sums of money. Recruitment was completed in August 2019 through a two-stage process. First, a public invitation to complete a screening survey was published on MTurk. Then, responding individuals who were 18 to 65 years old, lived in the United States, and had internet access were sent a private invitation to participate in the full study. In all, 1,876 individuals completed the screening survey, and 1,437 (76.6\%) met the eligibility criteria and were sent a private invitation. Of those, 766 (53.3\%) began the study survey, and complete data from 734 $(51.2 \%)$ were used in the analysis.

\section{Outcomes}

Individual alcohol use history was measured using the 10-item Alcohol Use Disorders Identification Test (AUDIT) (Saunders et al, 1993). Eight items used 5-point Likert scales ranging from never to 4 or more times a week ( 7 items) or 1 or 2 drinks to 10 or more drinks ( 1 item). These were coded from 0 to 4 . Two items used a 3-point Likert scale that included no, yes but not in the last year, and yes during the last year, which were coded 0,2 , and 4, respectively. All items were aggregated by summation $(\alpha=0.89)$. Alcohol cravings were measured using a 14-item version of the Desires for Alcohol Questionnaire (DAQ) (Love et al., 1998). All items used a 5point Likert scale ranging from strongly disagree to 
strongly agree, which were coded from 0 to 4. Responses were aggregated by summation to produce a total score $(\alpha=0.88)$ and for each DAQ sub-scale, which included desires and intentions to drink ( 6 items, $\alpha=0.86$ ), negative reinforcement ( 4 items, $\alpha=0.80$ ), control over drinking ( 2 items, $\alpha=0.68$ ), and mild desires to drink (2 items, $\alpha=0.92$ ). The control over drinking items were reverse coded.

\section{Qualitative Self-Reported Topography}

To self-report topography, participants were presented with a randomized list of 20 words, which were selected because they were thought to be synonymous or associated with the amount or speed of alcohol consumption (i.e., swill, drink, sample, shots, slug, swig, pull, guzzle, sip, down, nip, nurse, chug, gorge, inhale, bender, slurp, gulp, glug, swallow). Participants were instructed to select all the words that describe how they typically drink their usual alcoholic beverage. Selected items were coded as 1 . Nonselected items were coded as 0 .

\section{Covariates}

Several demographic and socioeconomic status (SES) variables were measured. Demographic variables include age, sex, race, ethnicity, and marital status. SES included income and education level.

\section{Statistical Analysis}

The distribution of all continuous variables were examined for skewness prior to analysis. Total AUDIT scores were positively skewed, and a square-root transformation was applied, which produced an approximately normal curve.

A latent class analysis (LCA) was conducted on self-reported topography to identify clustering of responses. There no were prior assumptions on the number of latent classes, and additional classes were added until model fit, as measured by the Akaike
Information Criterion (AIC) and the Bayesian Information Criterion (BIC), was minimized, and the classes remained substantively meaningful. Alcohol topography class, after adjustment for the covariates, was then used as the independent variable to predict AUDIT and DAQ scores in a linear regression analysis. Unadjusted and adjusted models, which controlled for all covariates, were specified.

The LCA models were specified using full maximum likelihood and were conducted using Mplus Version 8.3 (Los Angeles, CA: Muthén \& Muthén). Descriptive statistics and regression analysis were conducted with SPSS v26.0 (Armonk, NY: IBM Corp.). Statistical significance was set at 0.05 .

\section{Results}

A majority of the sample was female $(59.1 \%)$, White $(79.6 \%)$, and non-Hispanic (89.9\%) (Table 1). Participants were predominantly married or in a domestic partnership (52.7\%), with $39.1 \%$ single, never married, and $57.5 \%$ had a Bachelor's degree or higher. Approximately 46\% earned more than US $\$ 60,000$ per year while $11.4 \%$ earned less than US $\$ 20,000$ per year. Mean age was 35.9 years old $(\mathrm{SD}=10.5)$ (Table 2). Mean AUDIT score was $5.7(\mathrm{SD}=5.6)$, which is considered minimal risk, and 48 participants $(6.5 \%)$ had an AUDIT score of zero. Mean DAQ score was 16.8 (SD = 11.8).

A three-class LCA solution was accepted $(\mathrm{AIC}=8306.7, \mathrm{BIC}=8591.8)$ over the two-class $(\mathrm{AIC}=8415.9, \mathrm{BIC}=8604.4)$ and four-class $(\mathrm{AIC}=8226.0, \mathrm{BIC}=8607.7)$ solutions. Although the four-class solution had a lower AIC than the three-class solution, the BIC was higher, and the fourth class was not considered substantively meaningful as it contained only 5 participants. The drinking topography of Class 1 ( $\mathrm{n}=195,26.6 \%$ ) was characterized by the words sample, sip, and nurse (Table 3). Class 2 ( $\mathrm{n}=134$, $18.3 \%$ ) was characterized by the words shot, swig, swallow, gulp, bender, chug, down, glug, and gurzle. 
Table 1. Descriptive statistics of categorical and ordinal variables

\begin{tabular}{|c|c|c|}
\hline Variable & & n (\%) \\
\hline \multirow[t]{2}{*}{ Sex } & Female & $434(59.1)$ \\
\hline & Male & $300(40.9)$ \\
\hline \multirow[t]{9}{*}{ Race* } & American & $20(2.7)$ \\
\hline & Indian/Alaska Native & \\
\hline & Asian & $81(11.0)$ \\
\hline & $\begin{array}{l}\text { Black/African } \\
\text { American }\end{array}$ & $60(8.2)$ \\
\hline & Caucasian & $584(79.6)$ \\
\hline & Native & $3(0.4)$ \\
\hline & Hawaïan/Pacific & \\
\hline & Islander & \\
\hline & Some other race & $20(2.7)$ \\
\hline \multirow[t]{2}{*}{ Ethnicity } & Hispanic & $74(10.1)$ \\
\hline & Non-Hispanic & $89.9(660)$ \\
\hline \multirow{5}{*}{$\begin{array}{l}\text { Marital } \\
\text { status }\end{array}$} & Single, never married & $287(39.1)$ \\
\hline & $\begin{array}{l}\text { Married or domestic } \\
\text { partnership }\end{array}$ & $387(52.7)$ \\
\hline & Divorced & $43(5.9)$ \\
\hline & Widowed & $5(0.7)$ \\
\hline & Separated & $12(1.6)$ \\
\hline \multirow[t]{12}{*}{ Income } & Less than $\$ 10,000$ & $29(4.0)$ \\
\hline & $\$ 10,000$ to $\$ 19,999$ & $54(7.4)$ \\
\hline & $\$ 20,000$ to $\$ 29,999$ & $77(10.5)$ \\
\hline & $\$ 30,000$ to $\$ 39,999$ & $65(8.9)$ \\
\hline & $\$ 40,000$ to $\$ 49,999$ & $89(12.1)$ \\
\hline & $\$ 50,000$ to $\$ 59,999$ & 85 (11.6) \\
\hline & $\$ 60,000$ to $\$ 69,999$ & $71(9.7)$ \\
\hline & $\$ 70,000$ to $\$ 79,999$ & $60(8.2)$ \\
\hline & $\$ 80,000$ to $\$ 89,999$ & $39(5.3)$ \\
\hline & $\$ 90,000$ to $\$ 99,999$ & $47(6.4)$ \\
\hline & $\$ 100,000$ to $\$ 149,999$ & $88(12.0)$ \\
\hline & $\$ 150,000$ or more & $30(4.1)$ \\
\hline \multirow[t]{5}{*}{ Education } & Less than bigh school & $6(0.8)$ \\
\hline & $\begin{array}{l}\text { High school } \\
\text { degree/GED }\end{array}$ & $62(8.4)$ \\
\hline & Some college & $244(33.2)$ \\
\hline & Bachelor's degree & $313(42.6)$ \\
\hline & Master's degree or higher & $109(14.9)$ \\
\hline
\end{tabular}

*Will not add to $100 \%$ because participants were able to select more than 1 .

Class 3 ( $\mathrm{n}=405,55.2 \%$ ) predominantly used the words drink and sip, with the frequency of the remaining descriptors remaining low.
Table 2. Descriptive statistics of continuous variables

\begin{tabular}{lc}
\hline Variable & Mean (SD) \\
\hline Age & $35.9(10.5)$ \\
AUDIT Score & $5.7(5.6)$ \\
Total DAQ Score & $16.8(11.8)$ \\
DAQ Sub-Scales & \\
Desires and intentions to drink & $6.2(5.8)$ \\
$\quad$ Negative reinforcement & $4.8(4.2)$ \\
$\quad$ Control over drinking & $2.1(2.4)$ \\
$\quad$ Mild desires to drink & $3.6(2.6)$ \\
\hline
\end{tabular}

In the unadjusted and adjusted regression analysis, Class 1 was used as the referent group. In the unadjusted model, class assignment explained $18.6 \%$ of the variance in AUDIT scores, and AUDIT scores were significantly higher in Class $2(p<0.001)$ (Table 4), where the mean AUDIT score was 11.1 (SD $=7.2$ ), which is suggestive of hazardous or harmful alcohol consumption (Figure 1). AUDIT scores were not significantly different in Class $3(p=0.641)$. Similar results were obtained after adjusting for demographic and SES covariates ( plass $2_{2}<0.001$; p Class $3=0.909$ ).

Class assignment explained $19.2 \%$ of the variance in total DAQ scores in the unadjusted model, and DAQ scores were significantly higher in Class 2 ( $p<0.001)$ relative to Class 1 , but there was no difference with Class $3(\mathrm{p}=0.054)$ (Table 4; Figure 1). The results were similar in the adjusted model ( plass $2_{2}<0.001$; p Class $3_{3}=0.515$ ). Among DAQ sub-scales, scores were consistently higher across sub-scales in Class 2 relative to Class 1 (p's $<0.001$ to $\mathrm{p}=0.001$ ). Conversely, there was no significant differences in sub-scale scores between Class 1 and Class 3 except for the control over drinking sub-scale, where Class 3 scores were significantly greater ( $p$ $=0.004$.)

\section{Discussion}

The exploratory analysis suggests that self-reported alcohol topography may be associated with individual alcohol use history and alcohol cravings and may explain a substantial 
Table 3. Topography descriptor frequencies by latent class, $n(\%)$

\begin{tabular}{lcccc}
\hline Descriptor & Class 1 & Class 2 & Class 3 & Total \\
\hline Bender & $0(0.0)$ & $21(15.7)$ & $0(0.0)$ & $21(2.9)$ \\
Chug & $0(0.0)$ & $43(32.1)$ & $7(1.7)$ & $50(6.8)$ \\
Down & $0(0.0)$ & $33(24.6)$ & $3(0.7)$ & $36(4.9)$ \\
Drink & $115(59.0)$ & $97(72.4)$ & $201(49.6)$ & $413(56.3)$ \\
Glug & $0(0.0)$ & $19(14.2)$ & $0(0.0)$ & $19(2.6)$ \\
Gorge & $0(0.0)$ & $6(4.5)$ & $1(0.2)$ & $7(1.0)$ \\
Gulp & $0(0.0)$ & $56(41.8)$ & $19(4.7)$ & $75(10.2)$ \\
Guzzle & $0(0.0)$ & $33(24.6)$ & $3(0.7)$ & $36(4.9)$ \\
Inhale & $0(0.0)$ & $13(9.7)$ & $0(0.0)$ & $13(1.8)$ \\
Nip & $24(12.3)$ & $14(10.4)$ & $0(0.0)$ & $38(5.2)$ \\
Nurse & $134(68.7)$ & $17(12.7)$ & $21(5.2)$ & $172(23.4)$ \\
Pull & $2(1.0)$ & $19(14.2)$ & $5(1.2)$ & $26(3.5)$ \\
Sample & $94(48.2)$ & $19(14.2)$ & $10(2.5)$ & $123(16.8)$ \\
Shots & $11(5.6)$ & $64(47.8)$ & $60(14.8)$ & $135(18.4)$ \\
Sip & $195(100)$ & $72(53.7)$ & $218(53.8)$ & $485(66.1)$ \\
Slug & $1(0.5)$ & $14(10.4)$ & $0(0.0)$ & $15(2.0)$ \\
Slurp & $7(3.6)$ & $28(20.9)$ & $6(1.5)$ & $41(5.6)$ \\
Swallow & $58(29.7)$ & $71(53.0)$ & $65(16.0)$ & $194(26.4)$ \\
Swig & $41(21.0)$ & $59(44.0)$ & $27(6.7)$ & $127(17.3)$ \\
Swill & $6(3.1)$ & $18(13.4)$ & $0(0.0)$ & $24(3.3)$ \\
\hline
\end{tabular}

Table 4. Unadjusted and adjusted linear regression analysis to determine relationship between topographic latent class and AUDIT and DAQ scores.

\begin{tabular}{|c|c|c|c|c|c|c|c|}
\hline \multirow{3}{*}{\multicolumn{2}{|c|}{ Variable }} & \multirow{2}{*}{\multicolumn{3}{|c|}{ Unadjusted }} & \multirow{2}{*}{\multicolumn{3}{|c|}{ Adjusted }} \\
\hline & & & & & & & \\
\hline & & $\mathbf{b}$ & $95 \% \mathrm{CI}$ & $\mathrm{p}$ & b & $95 \% \mathrm{CI}$ & $\mathrm{p}$ \\
\hline \multirow[t]{3}{*}{$\overline{\text { AUDIT }}$} & Class 3 & 0.04 & $-0.13,0.22$ & 0.641 & -0.01 & $-0.19,0.17$ & 0.909 \\
\hline & Class 2 & 1.29 & $1.06,1.51$ & $<0.001$ & 1.15 & $0.91,1.38$ & $<0.001$ \\
\hline & Class 1 & & & & & & \\
\hline \multirow[t]{3}{*}{ DAQ } & Class 3 & 1.79 & $-0.03,3.61$ & 0.054 & 0.98 & $-0.84,2.80$ & 0.290 \\
\hline & Class 2 & 14.5 & $12.1,16.8$ & $<0.001$ & 12.9 & $10.5,15.3$ & $<0.001$ \\
\hline & Class 1 & . & , & . & . & . & 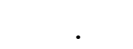 \\
\hline \multicolumn{8}{|l|}{ DAQ Sub-Scales } \\
\hline \multicolumn{2}{|c|}{ Desires and intentions to drink Class 3} & 0.73 & $-0.18,1.63$ & 0.115 & 0.30 & $-0.60,1.20$ & 0.515 \\
\hline & Class 2 & 6.77 & $5.61,7.93$ & $<0.001$ & 5.89 & $4.70,7.08$ & $<0.001$ \\
\hline & Class 1 & & & & . & & \\
\hline \multirow[t]{3}{*}{ Negative reinforcement } & Class 3 & 0.53 & $-0.14,1.20$ & 0.119 & 0.29 & $-0.39,0.86$ & 0.405 \\
\hline & Class 2 & 4.68 & $3.82,5.39$ & $<0.001$ & 4.27 & $3.38,5.15$ & $<0.001$ \\
\hline & Class 1 & & & & $\cdot$ & & \\
\hline \multirow[t]{3}{*}{ Control over drinking } & Class 3 & 0.61 & $0.20,1.01$ & 0.003 & 0.60 & $0.19,1.02$ & 0.004 \\
\hline & Class 2 & 0.97 & $0.45,1.49$ & $<0.001$ & 0.92 & $0.38,1.47$ & 0.001 \\
\hline & Class 1 & & & & & & \\
\hline \multirow[t]{3}{*}{ Mild desires to drink } & Class 3 & -0.07 & $-0.50,0.36$ & 0.746 & -0.21 & $-0.64,0.23$ & 0.350 \\
\hline & Class 2 & 2.08 & $1.53,2.62$ & $<0.001$ & 3.74 & $2.76,4.72$ & $<0.001$ \\
\hline & Class 1 & . & . & . & . & . & 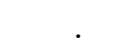 \\
\hline
\end{tabular}




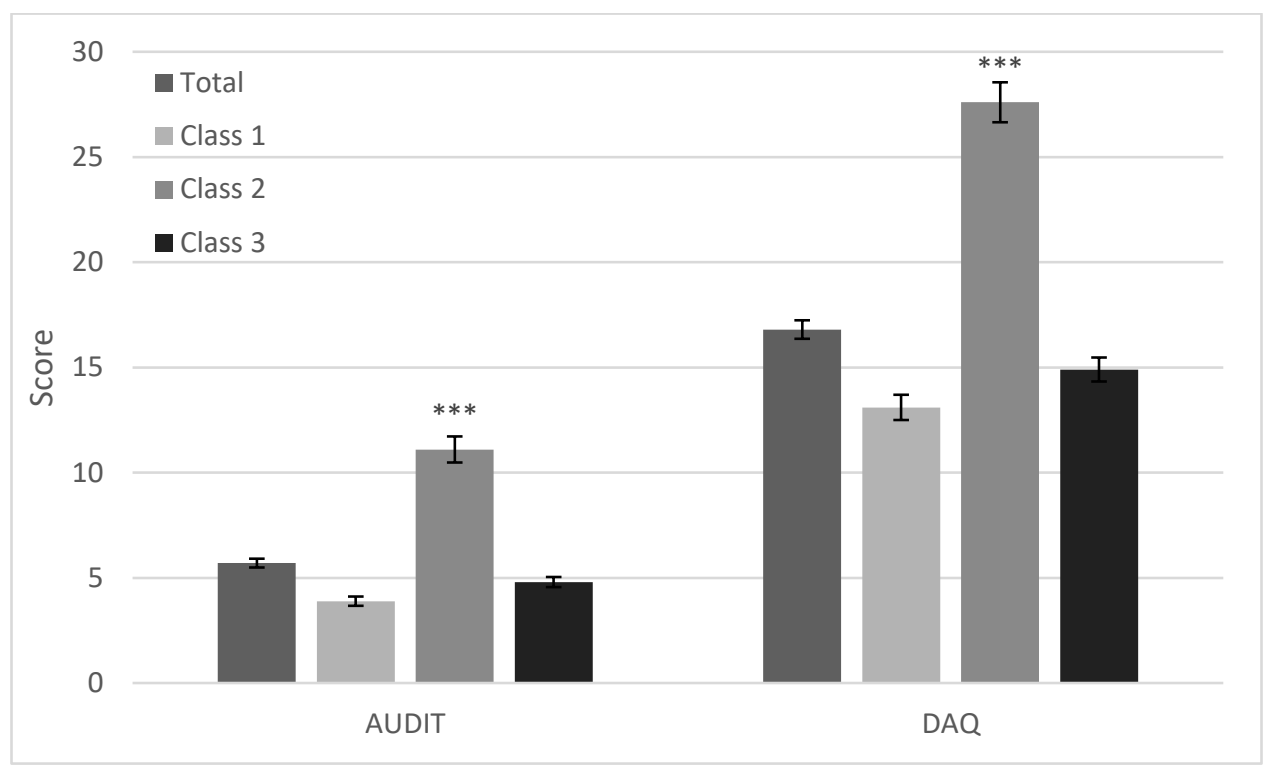

Figure 1. Total AUDIT and DAQ scores by latent class assignment with SE bars. ***Significantly different than Class 1 scores ( $\mathrm{p}<0.001)$.

proportion of the variance in these measures. Specifically, individuals who describe their drinking using words such as shot, swig, swallow, down, guzzle, and gulp may be more likely to be hazardous or harmful alcohol consumers and may have the highest levels of alcohol cravings. Those who only use the words drink and sip may have less control over their drinking once consumption begins, even though overall alcohol use risk is minimal. Although qualitative measures were used to measure alcohol topography, the findings are consistent with quantitative measures demonstrating that greater intensity of drinking is associated with higher estimated BAC levels, although further research is needed.

If the current findings are replicated, measures of alcohol topography may aid in identifying individuals at risk of alcohol use disorders and those whose disorder may be resistant to treatment. Indeed, the AUDIT was designed to identify individuals who have symptoms of alcohol dependence (Donovan et al., 2006), and the AUDIT consumption items plus an impaired control question accurately identified self-referred adults seeking alcohol treatment as either high or low severity cases (Wardell et al., 2020). Brief interventions may be more effective among individuals with higher AUDIT-C scores (Fernandez et al., 2019), and the AUDIT has been considered a useful tool in treatment planning and resource allocation (Niclasen et al., 2021). The DAQ is a reliable tool to measure alcohol cravings, and DAQ scores are strongly correlated with AUD severity as measured by the AUDIT (Pasche et al., 2013). Moreover, AUD severity may be influenced by deficits in the ability to regulate cue-induced craving (Naqvi et al., 2015), and the strength of an alcohol craving was associated with drinking in response to the craving among adults with an AUD (Dulin \& Gonzalez, 2017).

Interestingly, the descriptors that describe each of the three accepted latent classes mimic AUDIT risk levels and compare favorably to DAQ scores. The descriptors of Class 1 suggest minimal risk drinking, effective selfregulation while drinking, or even recent alcohol abstinence. The descriptors that characterize Class 2, which had consistently higher mean 
AUDIT and DAQ scores, may indicate a greater likelihood of binge or hazardous drinking and an inability to control drinking. Indeed, several descriptors suggestive of such drinking patterns, such as chug and guzzle, were predominantly selected by participants assigned to Class 2 . Finally, the descriptors of Class 3, which had very low frequencies of moderate terms, such as sample or nurse, and low frequencies of excessive consumption terms, suggest that these individuals are typically minimal risk drinkers but may occasionally drink excessively. Classifying drinkers in such a way is consistent with observations made during an alcohol selfadministration study in macaque monkeys (Baker et al., 2017). There, researchers noted that monkeys who gulped alcohol were more likely to be classified as very heavy drinkers at a 12-month follow-up while those who sipped alcohol were more likely to be light drinkers.

Measurements of alcohol topography may also be useful in determining the purpose or reason why a person consumes alcohol. For example, a person who sips or nurses an alcoholic beverage, which characterized Class 1, may be drinking for taste or primarily in social settings. In contrast, those who do shots or guzzle beverages may be purposefully seeking intoxication or self-medicating due to a comorbid condition, such as depression or anxiety. Several studies indicate that drinking motives mediate the relationship between non-addiction mental illness, such as attentive deficit hyperactivity disorder or post-traumatic stress disorder, and AUDs (Grazioli et al., 2019; Simpson et al., 2014). Others have concluded that alcohol use is a coping strategy, particularly among victims of sexual assault or intimate partner violence and persons living in high deprivation neighborhoods (Lindgren et al., 2012; Martin et al., 2019; Øverup et al., 2015).
Next Steps

Additional research is needed. First, validation studies are needed to determine the most appropriate method of measuring alcohol topography. Qualitative measures may be prone to recall and social desirability bias, and word lists used may need to be expanded to include words related to abstinence or spontaneous generated by participants themselves. Quantitative measures, such as biosensors, may not be able to distinguish between alcoholic beverage types, and video recording may be prone to the Hawthorne Effect, whereby drinking habits change because participants are being observed. Second, additional work is needed to determine whether alcohol topography is associated with health outcomes beyond estimated BAC. This work should incorporate quantity and frequency measures because if how someone drinks can predict AUDs and response to treatment regardless of drinking quantity or frequency, better targeting of prevention and treatment interventions may occur. Age and drinking context should also be considered.

\section{Limitations}

The study has several limitations. The sample was recruited from MTurk, which may be different than samples recruited using other means (Bunge et al., 2018), and the findings may not be generalizable to other populations, particularly non-English speakers. The sample list of words provided to participants to selfreport alcohol topography may be either incomplete or overly inclusive. Participants may also interpret the descriptors differently as definitions were not provided. Additional measures of alcohol consumption beyond the AUDIT, such as basic frequency and quantity measures, were not included. Self-report is also inherently subjective, and recall or social desirability bias may have occurred. However, self-reported topography did allow participants 
to consider their drinking styles for all types of alcoholic beverages.

\section{Conclusions}

Self-reported drinking topography may be associated with personal alcohol use history and alcohol cravings, although more research is needed. If the current findings are replicated, self-reported topography may be a useful tool to identify at-risk drinkers and those who may be resistant to treatment and other interventions.

\section{Acknowledgements}

This study was funded by the Rhode Island Foundation (\#2814_20190590). The funder had no role in the development, implementation, or reporting of this study.

\section{Conflicts of Interest}

The author declares there is no conflicts of interest.

\section{References}

Attwood, A. S., Scott-Samuel, N. E., Stothart, G., \& Munafò, M. R. (2012). Glass shape influences consumption rate for alcoholic beverages. PLoS One, 7, e43007.

Baker, E. J., Walter, N. A. R., Salo, A., Perea, P. R., Moore, S., Gonzalez, S., \& Grant, K. A. (2017). Identifying future drinkers: Behavioral analysis of monkeys initiating drinking to intoxication is predictive of future drinking classification. Alcoholism: Clinical and Experimental Research, 41, 626-636.

Bunge, E., Cook, H. M., Bond, M., Williamson, R. E., Cano, M., Barrera, A. Z., ..., Muñoz, R. F. (2018). Comparing Amazon Mechanical Turk with unpaid internet resources in online clinical trials. Internet Interventions, 12, 68-73.

Carpenter, R. W., Padovano, H. T., Emery, N. N., \& Miranda, R. (2019). Rate of alcohol consumption in the daily life of adolescents and emerging adults. Psychopharmacology, 236, 31113124.
Donovan, D. M., Kivlahan, D. R., Doyle, S. R., Longabaugh, R., \& Greenfield, S. F. (2006). Concurrent validity of the Alcohol Use Disorders Identification Test (AUDIT) and AUDIT zones in defining levels of severity among out-patients with alcohol dependence in the COMBINE study. Addiction, 101, 1696-1704. Dulin, P. L., \& Gonzalez, V. M. (2017). Smartphone-based, momentary intervention for alcohol cravings amongst individuals with an alcohol use disorder. Psychology of Addictive Behaviors, 31, 601-607.

Fernandez, A. C., Waller, R., Walton, M. A., Bonar, E. E., Ignacio, R. V., Chermack, S. T., ... Blow, F. C. (2019). Alcohol use severity and age moderate the effects of brief interventions in an emergency department randomized controlled trial. Drug and Alcohol Dependence, 194, 386-394.

Franken, F. H., Pickworth, W. B., Epstein, D. H., \& Moolchan, E. T. (2006). Smoking rates and topography predict adolescent smoking cessation following treatment with nicotine replacement therapy. Cancer Epidemiology, Biomarkers, and Prevention, 15, 154-157.

Grazioli, V. S., Gmel, G., Rougemont-Bücking, A., Baggio, S., Daeppen, J.-B., \& Studer, J. (2019). PLoS One, 14, e0218469.

Gunn, R. L., Steingrimsson, J. A., Merrill, J. E., Souza, T., \& Barnett, N. (2021). Characterising patterns of alcohol use among heavy drinkers: A cluster analysis utilising alcohol biosensor data. Drug and Alcohol Review. doi:10.1111/dar.13306. Hammond, D., Fong, G. T., Cummings, K. M., \& Hyland, A. (2005). Smoking topography, brand switching, and nicotine delivery: results from an in vivo study. Cancer Epidemiology, Biomarkers, and Prevention, 14, 1370-1375.

Leeman, R. F., Corbin, W. R., Nogueira, C., Krishnan-Sarin, S., Potenza, M. N., \& O’Malley, S. S. (2013). A human alcohol self-administration paradigm to model individual differences in impaired control over alcohol use. Experimental and Clinical Psychopharmacology, 21, 303-314. 
Leeman, R. F., Nogueira, C., Wiers, R. W., Cousijn, J., Serafini, K., DeMartini, K. S., ..., O’Malley, S. S. (2018). A test of multi-session automatic action tendency retraining to reduce alcohol consumption among young adults in the context of a human laboratory paradigm. Alcoholism: Clinical and Experimental Research, 42, 803-814.

Lindgren, K. P., Neighbors, C., Blayney, J. A., Mullins, P. M., \& Kaysen, D. (2012). Do drinking motives mediate the association between sexual assault and problem drinking? Addictive Behaviors, 37, 323-326.

Love, A., James, D., \& Willner, P. (1998). A comparison of two alcohol craving questionnaires. Addiction, 93, 1091-1102.

Martin, G., Inchley, J., \& Currie, C. (2019). Do drinking motives mediate the relationship between neighborhood characteristics and alcohol use among adolescents? International Journal of Environmental Research and Public Health, 16, 853.

Naqvi, N. H., Ochsner, K. N., Kober, H., Kuerbis, A., Feng, T., Wall, M., \& Morgenstern, J. (2015). Cognitive regulation of craving in alcohol dependent and social drinkers. Alcoholism: Clinical and Experimental Research, 39, 343-349.

Niclasen, B., Flyger, J., Becker, U., Nielson, B., \& Nielsen, A. S. (2021). Implementation of AUDIT in the treatment planning process for alcohol use disorder in Greenland. Nordic Journal of Psychiatry, 75, 145-151.

Øverup, C. S., Dibello, A. M., Brunson, J. A., Acitelli, L. K., \& Neighbors, C. (2015). Drowning the pain: Intimate partner violence, and drinking to cope prospectively predict problem drinking. Addictive Behaviors, 41, 152161.
Pasche, S. C., Garner, M., Baldwin, D. S., \& Sinclair, J. M. A. (2013). "Craving": exploring the components of the Desires for Alcohol Questionnaire (DAQ) and the relation to the severity of alcohol problems. Journal of Studies on Alcohol and Drugs, 74, 950-955.

Pickworth, W. B., Rosenberry, Z. R., O’Grady, K. E., \& Koszowski, B. (2017). Dual use of cigarettes, little cigars, cigarillos, and large cigars: Smoking topography and toxicant exposure. Tobacco Regulatory Science, 3, S72-S83.

Roberts, W., \& McKee, S. A. (2019). Mobile alcohol biosensors and pharmacotherapy development research. Alcohol, 81, 149-160.

Saunders, J. B., Aasland, O. G., Babor, T. F., de la Fuente, J. R., \& Grant, M. (1993). Development of the Alcohol Use Disorders Identification Test (AUDIT): WHO collaborative project on early detection of persons with harmful alcohol consumption-II. Addiction, 88, 791-804.

Simpson, T. L., Stappenbeck, C. A., Luterek, J. A., Lehavot, K., \& Kaysen, D. L. (2014). Drinking motives moderate daily relationships between PTSD symptoms and alcohol use. Journal of Abnormal Psychology, 123, 237-247.

Troy, D. M., Attwood, A. S., Maynard, O. M., Scott-Samuel, N. E., Hickman, M., Marteau, T. M., \& Munafò, M. R. (2017). Effect of glass markings on drinking rate in social alcohol drinkers. European Journal of Public Health, 27, 352356.

Wardell, J. D., Cunningham, J. A., Quilty, L. C., Carter, S., \& Hendershot, C. S. (2020). Can the AUDIT consumption items distinguish lower severity from high severity patients seeking treatment for alcohol use disorder? Journal of Substance Abuse Treatment, 114, 108001. 